OPEN ACCESS

Edited by:

Kay Tetzlaff,

University Hospital of Tübingen,

Germany

Reviewed by:

Costantino Balestra,

Haute École Bruxelles-Brabant

(HE2B), Belgium

Danilo Cialoni,

Dan Europe Foundation, Italy

François Guerrero,

Université de Bretagne Occidentale

France

${ }^{*}$ Correspondence:

Ran Arieli

arieli1940@gmail.com

Specialty section:

This article was submitted to Environmental, Aviation and Space

Physiology,

a section of the journal

Frontiers in Physiology

Received: 30 August 2021 Accepted: 21 September 2021

Published: 15 October 2021

Citation:

Arieli R (2021) Endothelial Injury in Diving: Atomic Force-, Electronicand Light-Microscopy Study of the

Ovine Decompressed Blood Vessels.

Front. Physiol. 12:767435.

doi: 10.3389/fphys.2021.767435

\section{Endothelial Injury in Diving: Atomic Force-, Electronic-, and Light-Microscopy Study of the Ovine Decompressed Blood Vessels}

\author{
Ran Arieli ${ }^{1,2 *}$ \\ ${ }^{1}$ Israel Naval Medical Institute, Israel Defense Forces Medical Corps, Haifa, Israel, ${ }^{2}$ Eliachar Research Laboratory, Western \\ Galilee Medical Center, Nahariya, Israel
}

We suggested that the nanobubbles, which appear at the active hydrophobic spots (AHSs) at the luminal aspect of the blood vessels, are the gas micronuclei from which the decompression bubbles evolve and the endothelial injury during the decompression is due to the tearing off the cell membranes with the detaching bubbles. Ovine blood vessels were stretched over the polycarbonate plates or glass microscopic slides and were exposed under saline to the hyperbaric pressure $(1,013 \mathrm{kPa}, 19 \mathrm{~h})$. Following decompression, the blood vessels were photographed for the identification (by bubble formation) of the AHS. Nanobubbles could not be demonstrated at the AHS by using the atomic force microscopy (AFM) because of the roughness of the surface, which disabled the close contact of the probe. In the electron microscopy, no endothelial cells were observed in the samples from the area near to the AHS, but the underlying elastin layer of the intima was observed adjacent to the media. Some intact endothelial cells were observed only in the locations far from an AHS. In the optical microscopy, no endothelial cells were observed in the blood vessels in close proximity to the AHS and in some sections, debris or a detached cluster of the endothelial cells were observed. Intact endothelial cells could be found at the sites distant from an AHS. This study supports the assumption, where the detached bubbles tear off the endothelial cells and cause the initial endothelial injury following the decompression.

\section{Keywords: active hydrophobic spot AHS, bubbles, detachment, nanobubble, endothelium}

\section{INTRODUCTION}

In the search for the hypothesized gas micronuclei from which the bubbles evolve during the decompression after diving, we succeeded in establishing the chain of the events (Arieli, 2017). The lung surfactant such as dipalmitoylphosphatidylcholine (DPPC) leaks into the bloodstream. By leaving the plasma, the DPPC settles on the luminal aspect of the blood vessels to create an oligolamellar lining of the phospholipids (Hills, 1992). We named this site an "active hydrophobic spot" (AHS). The nanobubbles formed at the AHS, regardless of the diving activity, from the dissolved gas, become the gas micronuclei from which the bubbles evolve on the decompression. Decompression bubbles detach from the AHS at a force of $4.5 \times 10^{-6} \mathrm{~N}$, which is lower than the adhesion hydrophobic force of $38 \times 10^{-6} \mathrm{~N}$. In addition to the other 
considerations (Arieli and Marmur, 2017), led us to suggest that a bubble detaches with a piece of the underlying membrane. The adhesion force between the membrane bilayer is low and may cavitate by exposure to the clinical ultrasound (Krasovitski et al., 2011). Thus, this study related the endothelial injury during the decompression to the tearing off of the cell membranes with the detaching bubbles. This mechanism is not surprising, since it is well accepted to flush bubbles for descementing the endothelial cells for the preparation of the cell culture or implantation (Ruzza et al., 2015). Endothelial injury during the decompression is well established and was even suggested as the main cause of the decompression illness (DCI) (Madden and Laden, 2009; Madden et al., 2010; Lambrechts et al., 2013; Zhang et al., 2016, 2017a,b). The aim of this study was to delve into the microworld of the bubbles and the endothelial cells in the blood vessels of the sheep.

\section{RESEARCH DESIGN AND METHODS}

Initially, an effort was made to watch the nanobubbles, which were supposed to form at the AHS with the aid of the atomic force microscopy (AFM). Nanobubbles were detected at any tested artificial hydrophobic surface underwater, but not yet at the hydrophobic animated tissue. The second phase was to observe the damage to the endothelial cells by detaching the bubbles by using the electron microscopy (EM). This study expected to observe the breaches in the AHS oligolamellar layer. In the third phase, this study observed the sections from the blood vessels at the AHS and controls the samples by using light microscopy (LM). Each phase of the study was dictated by the previous one.

\section{Tissue Preparation Common to the Three Phases}

The methods were described in detail in the previous studies (Arieli and Marmur, 2016; Arieli et al., 2016, 2019) and will be presented briefly here.

The complete heart and lungs from 10 slaughtered sheep (harvested on separate days) were obtained at the abattoir. In the laboratory, under the saline and without any exposure to air, this study sampled the four blood vessels such as the aorta, superior vena cava, pulmonary vein, and pulmonary artery. For the AFM study, the blood vessels were clamped on both sides denying contact to air from the interior. They were then extracted from the saline and the external surface was wiped dry with the filter paper. Two polycarbonate plates of $2.5 \mathrm{~cm} \times 2.5 \mathrm{~cm}$ were glued together by using cyanoacrylate adhesive. Then, under saline, the two plates were cut free. For the electron and light microscopy, the blood vessels were gently stretched on the microscope slides held by the metal clips with the luminal aspect exposed. Eight polycarbonate plates or four slides were placed anaerobically at the bottom of a rectangular Pyrex vessel $(26 \mathrm{~cm} \times 17 \mathrm{~cm}$, height $5 \mathrm{~cm}$ ) under $2.5 \mathrm{~cm}$ of saline.

The rectangular Pyrex vessel containing the blood vessels was transferred to a 150-L hyperbaric chamber and placed on a pair of the welded aluminum plates, with cooled water circulating in the space between the two plates. Cooling during the hyperbaric exposure was necessary for the tissue preservation.
The temperature of the circulating water was $6^{\circ} \mathrm{C}$. Saline temperature was $1^{\circ} \mathrm{C}$ compared to the circulating water. Pressure was elevated (by using air) at a rate of $200 \mathrm{kPa} / \mathrm{min}$ to $1,013 \mathrm{kPa}$, $90 \mathrm{~m}$ sea water, and remained at that pressure overnight $(\sim 19 \mathrm{~h})$. In the following morning, the chamber was decompressed at a rate of $200 \mathrm{kPa} / \mathrm{min}$. The rectangular vessel was placed carefully on a heating pad (E12107819, Sunbeam, Hong Kong, China) for photography. Saline was gently mixed to let the upper, gas-rich layer, mix with the deeper layers. This study began automated photographing at 5-s intervals, $15 \mathrm{~min}$ after the end of the decompression, for a period of $30 \mathrm{~min}$ (appropriate for the detection of the AHS) (Arieli and Marmur, 2016) for the AFM protocol and $60 \mathrm{~min}$ (allowing more time for the detachment of the bubbles) for the other two protocols. At the end of this photographic session, the rectangular vessel was kept in the refrigerator. Active hydrophobic spots can be determined by observing the formation of the bubbles after the decompression.

The photographs of each sample were examined in sequence for the appearance of the bubbles. At the end of the observation, the location of the AHS and the number of bubbles produced at each AHS were noted on the photograph that had been prepared for use during tissue sampling. Both locations of the AHS and control (area distanced from an AHS) were marked on the photograph to be sampled for the microscopy. For a control for the LM in order to exclude the effect of the detaching bubbles and prolonged hyperbaric exposure, blood vessels were prepared as previously described and under saline (without hyperbaric exposure), it was taken for the LM sampling.

\section{METHODS AND RESULTS}

\section{Atomic Force Microscopy Study}

In this study, we aimed to observe the nanobubbles at the AHS.

In the day following decompression, the rectangular vessel with the blood vessels under saline was transferred to the AFM department in Weizmann Institute of Science, Rehovot, Israel.

Measurements were made in a JPK NanoWizard 3 AFM by using the qp-BioAC-CI probes from the Nanoworld. Custom cells were prepared to house the sample and the liquid while fitting the dimensions of the AFM. The AFM topographical measurements were attempted in the two different modes. The first mode is the AC mode, where the probe is excited at its resonance frequency and the amplitude is controlled, as it taps on the surface by a feedback, which provides the height changes, i.e., topography during the scan. The second mode is the QI mode where the probe is also oscillated, but not at the resonance frequency, but rather at a lower rate, two to three orders of the magnitude slower. In this case, not the amplitude, but the peak force applied on the sample is controlled to get the topography. The QI mode was found to have better control over the dynamics and made it possible to acquire some images. The plates moved around within the saline-containing cell, but it was possible to fix them in place by using an underwater epoxy. After this, some images could be obtained but they were of poor quality because the sample is extremely rough and has loose tissue and debris on the surface. A further attempt after storing the sample in the refrigerator for 

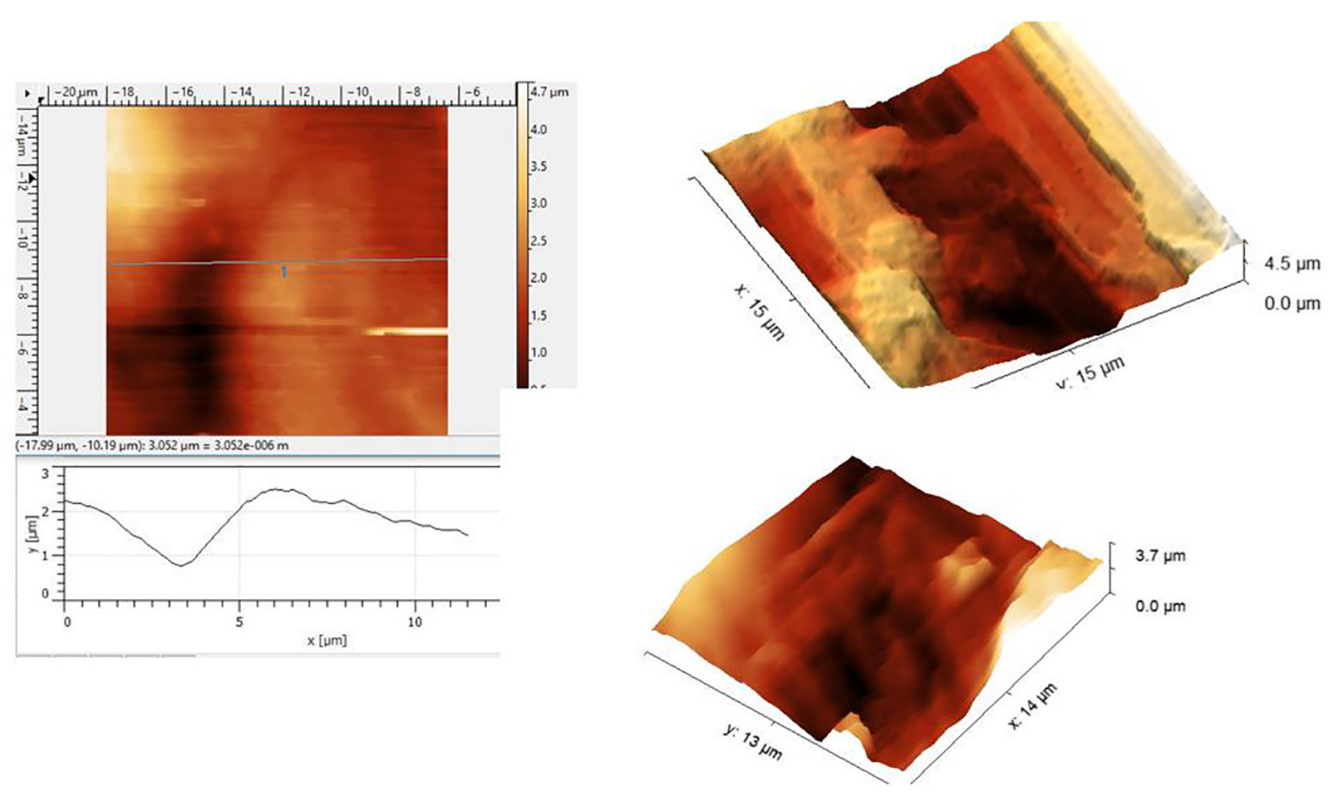

FIGURE 1 | Atomic force microscopy (AFM) scans under the saline of the luminal aspect of the blood vessels at the active hydrophobic spot (AHS) sites. Rough surface disables a closer approach to the sensing probe.

a couple of days was futile because the amount of debris in the saline solution had increased and the AFM signal (which is based on optical reflection) was not stable, even when the probe was not touching the surface.

A few representative images are given in Figure 1 showing the roughness of the surface. The roughness should be significantly smaller than the expected $100 \mathrm{~nm}$ size of the nanobubbles. The optical images are at a very large scale relative to the scanning size of the tens of microns. Nanobubbles could not be demonstrated

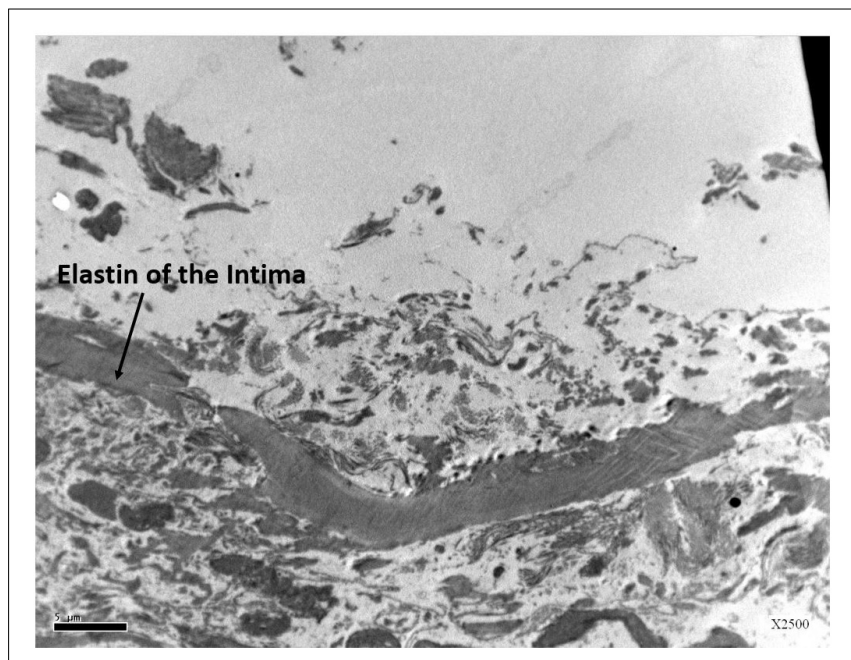

FIGURE 2 | Electron microscopy view of the luminal aspect of the blood vessels at the AHS sites. Only the elastin layer remained from the intima where the endothelial cells are missing. because of the roughness and the debris at the AHS. Therefore, the observation of the control area was of no use. The failure of the AFM attempt led us to continue with the electron microscopy study to look into the roughness of the surface and possible breaches in the AHS.

\section{Electron Microscopy Study}

The aim of the EM study was to observe the damage to the endothelial cell caused by the detaching bubble. This study expected to observe the breaches in the oligolamellar phospholipids (AHS) at the outer membrane of the endothelial cell. Blood vessels were extracted from the saline for direct sampling. Samples from the areas near the AHS and the locations distant from an AHS were dissected from the blood vessels of the sheep and were prefixed with $2.5 \%$ glutaraldehyde in 0.1 $\mathrm{M}$ phosphate buffer (PB) ( $\mathrm{pH} 7.4$ ) for $1 \mathrm{~h}$ and postfixed with $1 \%$ osmium tetroxide in $0.1 \mathrm{M} \mathrm{PB}$ for $1 \mathrm{~h}$. The specimens were routinely dehydrated by passing the tissue through a series of solutions with the increasing ethanol concentrations and then embedded in the Epon 812 epoxy resin. To examine the specimens, the thick Epon sections were first cut at $0.5-\mu \mathrm{m}$ thickness and routinely stained with toluidine blue. Then, the ultrathin sections were cut at 70-80-nm thickness with a diamond knife on an ultramicrotome and mounted on $\Phi 1 \mathrm{~mm} \times 2 \mathrm{~mm}$ single-slit copper carbon grids. The ultrathin sections of the tissues on the copper grids were double stained with uranyl acetate and lead citrate and, finally, observed under a JEOL 1011 transmission electron microscope (JEOL Ltd., Akishima, Tokyo, Japan) at an accelerating voltage of $80 \mathrm{kV}$.

This study expected to observe the breaches in the oligolamellar lining of the phospholipids at the AHS. However, 


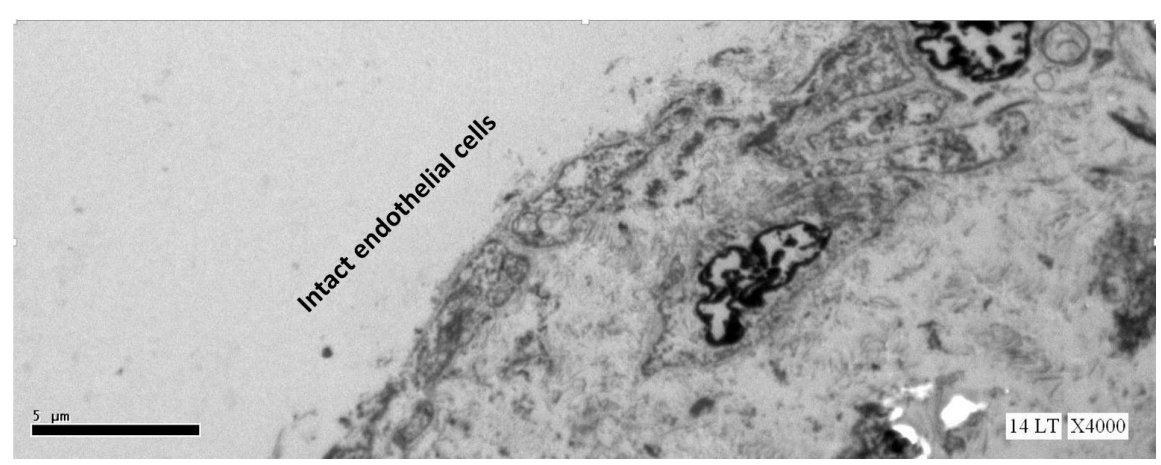

FIGURE 3 | Electron microscopy view of the luminal aspect of the blood vessels at a control site (away from the AHS). Intact endothelial cells are seen.

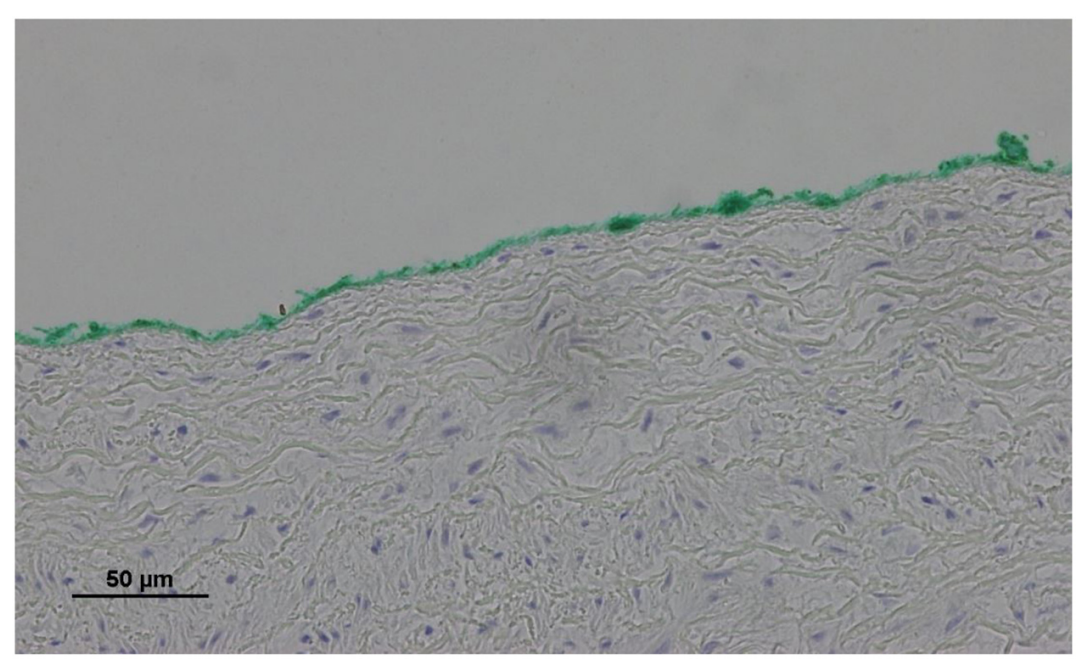

FIGURE 4 | Light microscopy view of the luminal aspect of the aorta at the AHS. Green line denotes the luminal aspect. There are no endothelial cells.

in the first few samples from the AHS, no endothelial cells were seen at the luminal side of the blood vessel. For denial of the wrong sampling, this study let the professional staff at the pathology department to make the sampling. Again, no endothelial cells were observed, but the elastin layer of the intima adjacent to the media was observed at the AHS (Figure 2) with some debris from the teared endothelial layer. Intact endothelial cells were observed only in the samples of the control area, distant from the AHS (Figure 3). Detaching bubbles teared off the entire endothelial layer at the AHS. Detachment of an entire endothelial layer led us to turn to observation with the LM.

\section{Light Microscopy Study}

Blood vessels were extracted from the saline for direct sampling. Samples from the areas near the AHS and sites distant from the defined AHS were dissected from the blood vessels of the sheep and the samples from the blood vessels were prepared in the same way without exposure to the hyperbaric conditions. The luminal aspect of the blood vessel, exposed to the hyperbaric chamber, was stained green for later identification.
For the observation of the endothelial cells, a typical marker CD31 was stained. CD31-specific immunohistochemistry (IHC) (clone JC/70A) was performed on 3- $\mu \mathrm{m}$ thick paraffin sections of the tissue blocks by using the Ventana BenchMark XT automated slide-staining system (Ventana Medical Systems Incorporation, Tucson, Arizona, United States) as recently described: After the cell conditioning with the cell conditioner 1 (Ventana Medical Systems Incorporation, Tucson, Arizona, United States) for $32 \mathrm{~min}$ and the preprimary peroxidase inhibition, the slides were incubated with the commercially available antibodies (dilution 1:70; Thermo Fisher Scientific, Kalamazoo, Michigan, United States) for $32 \mathrm{~min}$ at $37^{\circ} \mathrm{C}$. Primary antibodies were detected by using the iView $\mathrm{DAB}$ IHC Detection Kit (Ventana Medical Systems Incorporation, Tucson, Arizona, United States). Slides were counterstained with hematoxylin for 2 min each. Blood vessels, which were not exposed to the hyperbaric conditions, were stained with the standard H\&E.

No endothelial cells were observed in the hyperbaric exposed blood vessels at the areas near the AHS (Figure 4). In some sections, debris or a detached cluster of the endothelial cells were 

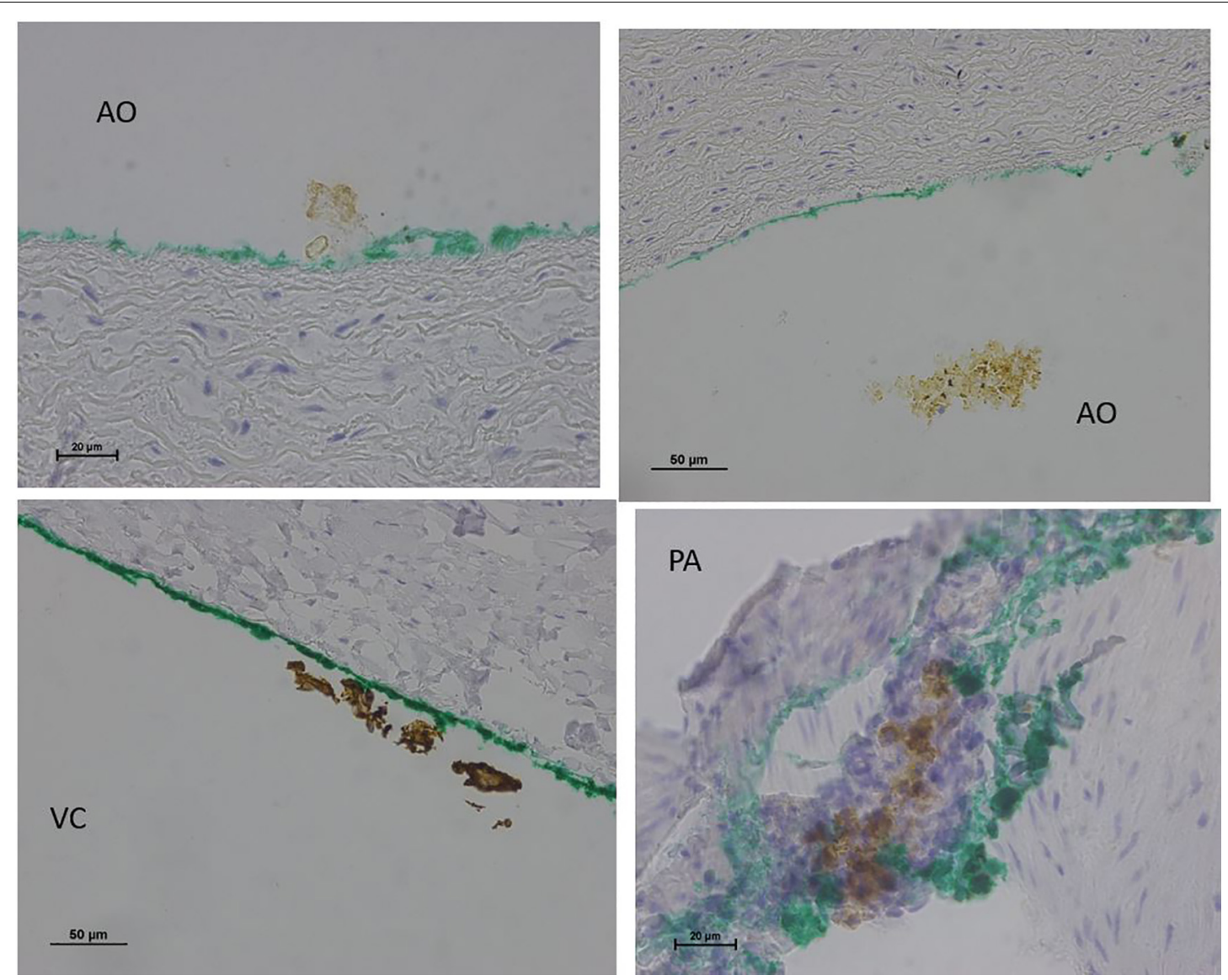

FIGURE 5 | Light microscopy view of the luminal aspect of the blood vessels: aorta-AO, superior vena cava-VC, and pulmonary artery-PA at the AHS. Green line denotes the luminal aspect. There is debris or detached cluster of the endothelial cells (brown color).

observed (Figure 5). Intact endothelial cells could be found at the sites distant from an AHS (Figure 6). Intact endothelial cells were observed in the control unexposed blood vessels (Figure 7).

\section{DISCUSSION}

In this study, the main outcome is that the detached bubble at the AHS tears off the entire underlying endothelial layer. Subsequently, it causes endothelial damage. The rough surface, which remained after $75 \mathrm{~min}$ of the detaching bubbles, is inaccessible to the close approach of the AFM probe. Thus, our previous suggestion (Arieli, 2017) that the bubble detaches from the AHS with a section of the endothelial membrane is supported. A suggestion similar to this study was made by the Madden and Laden (2007): Gas bubbles released into the circulation will interact with the surface of the vascular endothelium and may give rise to a measurable response linked to the state of the endothelium. The only difference is that this study considers the local (and not circulated) bubbles at the endothelium.

Klinger et al. (2011) presented an injury in the contact of the bubbles with the umbilical endothelium. They also showed that the addition of the surfactant prevented the damage. It is well possible that the hydrophobic tail of the surfactant clings to the hydrophobic AHS and, thus, the hydrophilic head of the surfactant prevents the attachment of the bubbles and the affected injury. Sobolewski et al. (2011) found a reduction in the endothelial injury when surfactant was added before the contact of an air bubble with a single endothelial cell. Surfactant also reduced the bubble adhesion in the arterioles and the damage produced with the injected microbubbles (Suzuki et al., 2004).

Thom et al. $(2018,2019)$ suggested that the microparticles induce the vascular injury. However, Lambrechts et al. (2017) proved that the vascular dysfunction is related to the decompression bubbles and not to the same diving protocol almost without the decompression bubbles (predive vibrations). Microparticles may induce further injury, but the initial injury is related to the detached bubbles. Zhang et al. (2017b) presented a high correlation between the markers of the endothelial injury and the total amount of the bubbles and the time course of the bubbles in the decompressed mice. Endothelial markers and bubble score peaked at about half an hour and the markers outlasted the bubbles $(<2 \mathrm{~h})$ at $12-24 \mathrm{~h}$. The good correlation suggests a cause-and-effect relationship and the outlasting endothelial injury points to further damage, 


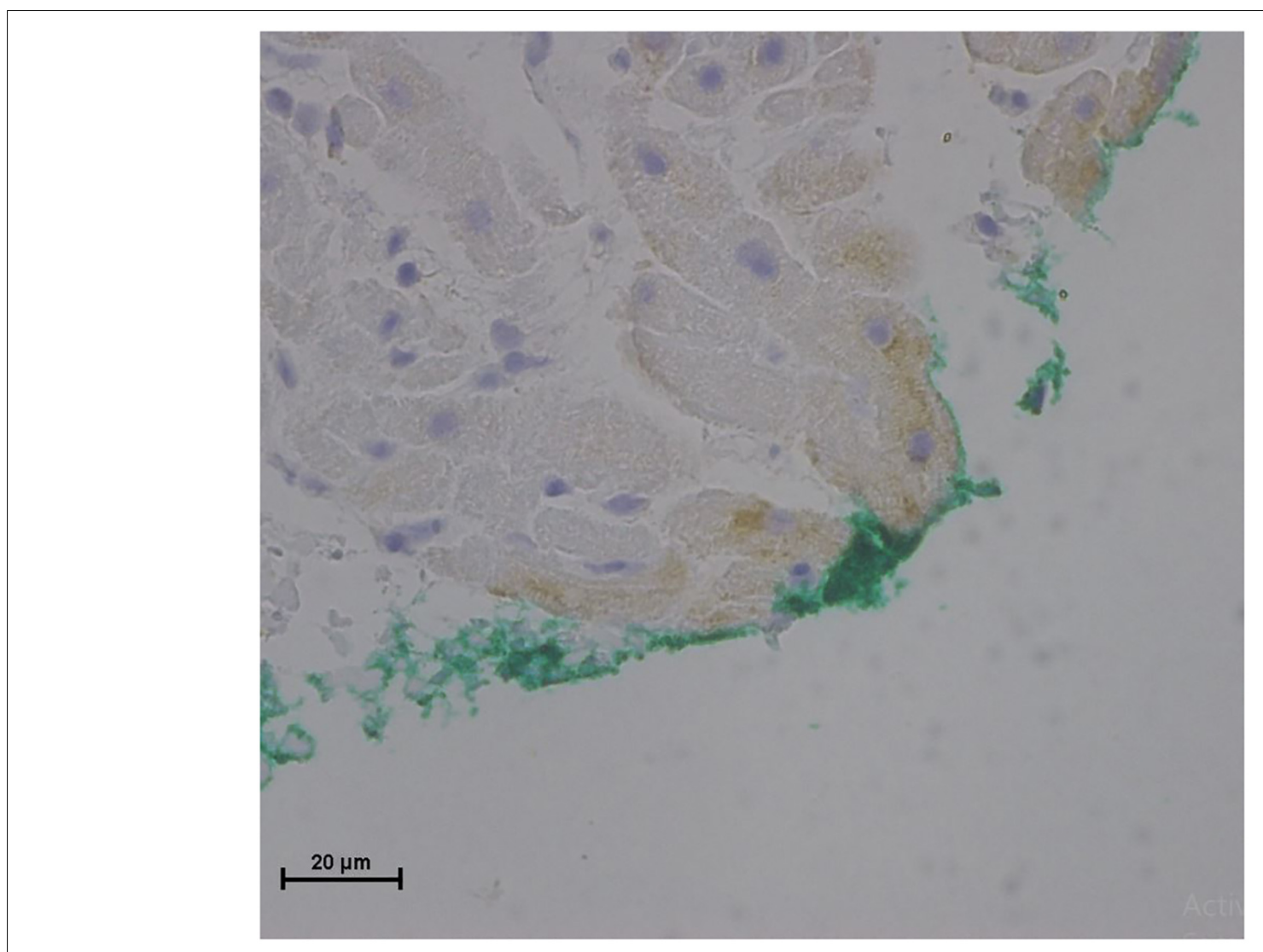

FIGURE 6 | Light microscopy view of the luminal aspect of the superior vena cava away from the AHS. Green line denotes the luminal aspect. There are intact endothelial cells.

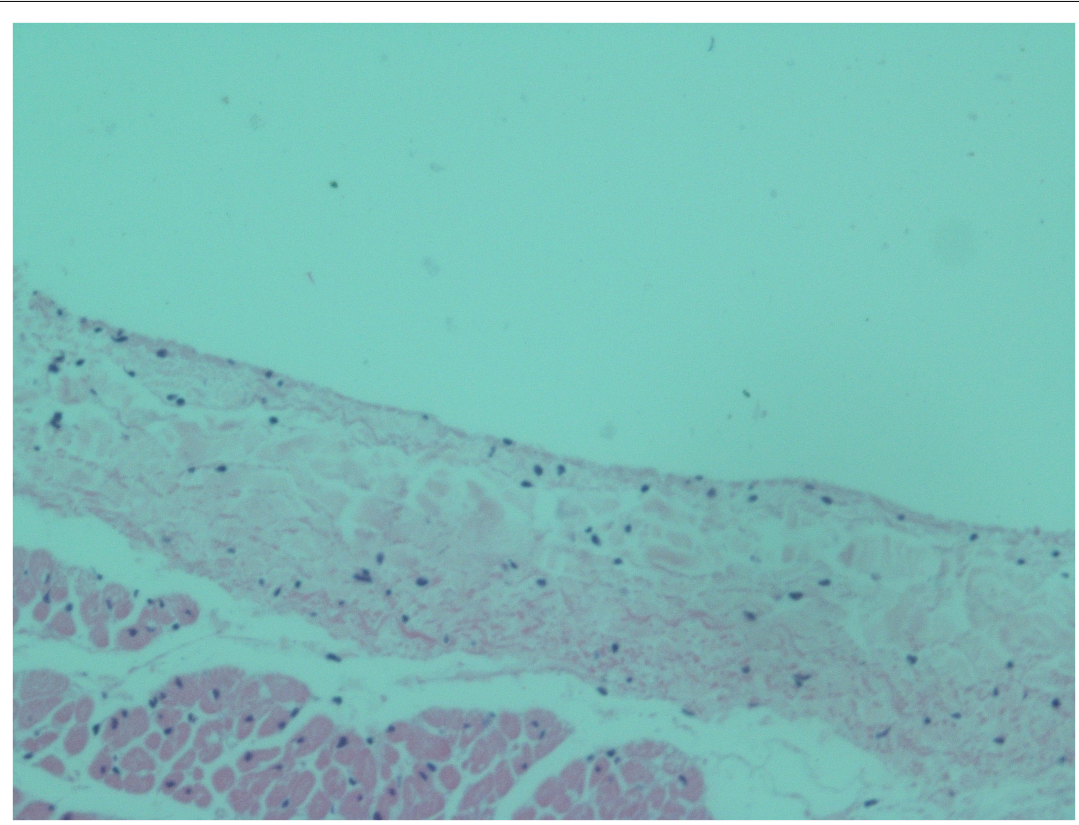

FIGURE 7 | Light microscopy view of the luminal aspect of the unexposed superior vena cava. There are intact endothelial cells. 
which may be induced by the microparticles. Nossum et al. (1999) exposed the pigs to high pressure. Endothelial damage, expressed as the total response of the pulmonary artery to the substance $\mathrm{P}$, was reduced only in the pigs with the recorded bubbles in the pulmonary artery, but not in the others. In their recent study, Yu et al. (2017) showed that the bubbles induced the endothelial microparticles in the cultured pulmonary endothelial cells from the rat that further caused the endothelial injury in both the cultured endothelial cell and the whole animal.

This study supports the assumption where the detached bubbles tear off the endothelial cells and cause the initial endothelial injury and agree with the conclusion of Zhang et al. (2017b): bubbles are the most likely initial causative agents of the endothelial dysfunction following the diving decompression.

Many of the manifestations of the DCI were related to the endothelial microparticles (Thom et al., 2011, 2012, 2013; Yang et al., 2012). We suggested that a detached venous bubble carries with it the endothelial membrane and after releasing most of its gas in the lung, it becomes a gas-containing endothelial microparticle (Arieli, 2017). This study suggests that the endothelial membranes are carried with the detached bubbles, which at least support the first step in the origin of the decompression microparticles.

\section{REFERENCES}

Arieli, R. (2017). Nanobubbles form at active hydrophobic spots on the luminal aspect of blood vessels: consequences for decompression illness in diving and possible implications for autoimmune disease - an overview. Front. Physiol. 8:591. doi: 10.3389/fphys.2017.00591

Arieli, R., Khatib, S., and Vaya, J. (2016). Presence of dipalmitoylphosphatidylcholine from the lungs atthe active hydrophobic spots in the vasculature where bubbles are formed on decompression. J. Appl. Physiol. 121, 811-815. doi: 10.1152/japplphysiol.00649.2016

Arieli, R., Khatib, S., and Vaya, J. (2019). Ovine plasma dipalmitoylphosphatidylcholine does notPredict decompression bubbling. Respir. Physiol. Neurobiol. 259, 26-29. doi: 10.1016/j.resp.2018.06.013

Arieli, R., and Marmur, A. (2016). Expansion of bubbles under a pulsatile flow regime in decompressed ovine blood vessels. Respir. Physiol. Neurobiol. 222, 1-5. doi: 10.1016/j.resp.2015.11.010

Arieli, R., and Marmur, A. (2017). A biophysical vascular bubble model for devising decompression procedures. Physiol. Rep. 5:e13191. doi: 10.14814/phy2. 13191

Hills, B. A. (1992). A hydrophobic oligolamellar lining to the vascular lumen in some organs. Undersea Biomed. Res. 19, 107-120.

Klinger, A. L., Pichette, B., Sobolewski, P., and Eckmann, D. M. (2011). Mechanotransductional basis of endothelial cell response to intravascular bubbles. Integr. Biol. 3, 1033-1042. doi: 10.1039/clib00017a

Krasovitski, B., Frenkel, V., Shoham, S., and Kimmel, E. (2011). Intramembrane cavitation as a unifying mechanism for ultrasound-induced bioeffects. Proc. Natl. Acad. Sci. U.S.A. 108, 3258-3263.

Lambrechts, K., Balestra, C., Theron, M., Henckes, A., Galinat, H., Mignant, F., et al. (2017). Venous gas emboli are involved in post-dive macro, but not microvascular dysfunction. Eur. J. Appl. Physiol. 117, 335-344. doi: 10.1007/ s00421-017-3537-9

Lambrechts, K., Pontier, J.-M., Mazur, A., Buzzacott, P., Morin, J., Wang, Q., et al. (2013). Effect of decompression-induced bubble formation on highly trained divers microvascular function. Physiol. Rep. 1:e00142. doi: 10.1002/ phy2.142

\section{DATA AVAILABILITY STATEMENT}

The raw data supporting the conclusions of this article will be made available by the authors, without undue reservation.

\section{AUTHOR CONTRIBUTIONS}

The author confirms being the sole contributor of this work and has approved it for publication.

\section{FUNDING}

This study was supported in part by the grant from the IDF Medical Corps and the Israel Ministry of Defense (MOD) (no 4440921551).

\section{ACKNOWLEDGMENTS}

We thank P. Brymann for the skillful editing of the manuscript and I. Minkov, Laboratory Manager, Pathology, Rambam Health Care Campus, Haifa, Israel for the excellent service in the microscopy.

Madden, L. A., Chrismas, B. C., Mellor, D., Vince, R. V., Midgley, A. W., McNaughton, L. R., et al. (2010). Endothelial function and stress response after simulated dives to $18 \mathrm{msw}$ breathing air or oxygen. Aviat. Space Environ. Med. 81, 41-45.

Madden, L. A., and Laden, G. (2007). Endothelial microparticles in vascular disease and as a potential marker of decompression illness. Eur. J. Underwater Hyperb. Med. 8, 6-10.

Madden, L. A., and Laden, G. (2009). Gas bubbles may not be the underlying cause of decompression illness - Theat-depth endothelial dysfunction hypothesis. Med. Hypotheses 72, 389-392. doi: 10.1016/j.mehy.2008.11.022

Nossum, V., Koteng, S., and Brubakk, A. O. (1999). Endothelial damage by bubbles in the pulmonary artery of the pig. Undersea Hyperb. Med. 26, 1-8.

Ruzza, A., Parekh, M., Salvalaio, G., Ferrari, S., Camposampiero, D., Amoureux, M.-C., et al. (2015). Bubble technique for descemet membrane endothelial keratoplasty tissue preparation in an eye bank: air or liquid? Acta Ophthalmol. 93, e129-e134. doi: 10.1111/aos.12520

Sobolewski, P., Kandel, J., Klinger, A. L., David, M., and Eckmann, D. M. (2011). Air bubble contact with endothelial cells in vitro induces calcium influx and IP3-dependent release of calcium stores. Am. J. Physiol. Cell Physiol. 301, C679-C686. doi: 10.1152/ajpcell.00046.2011

Suzuki, A., Armstead, S. C., and Eckmann, D. M. (2004). Surfactant reduction in embolism bubble adhesion and endothelial damage. Anesthesiology 101, 97-103. doi: 10.1097/00000542-200407000-00016

Thom, S. R., Bhopale, V. N., and Yang, M. (2019). Microparticle-induced vascular injury in mice following decompression is inhibited by hyperbaric oxygen: effects on microparticles and interleukin-1ß. J. Appl. Physiol. 126, 1006-1014. doi: 10.1152/japplphysiol.01109.2018

Thom, S. R., Bhopale, V. N., Yu, K., and Yang, M. (2018). Provocative decompression causes diffuse vascular injury in mice mediated by microparticles containing interleukin-1ß. J. Appl. Physiol. 125, 1339-1348. doi: 10.1152/japplphysiol.00620.2018

Thom, S. R., Milovanova, T. N., Bogush, M., Bhopale, V. M., Yang, M., Bushmann, K., et al. (2012). Microparticle production, neutrophil activation, and intravascular bubbles following open-water SCUBA diving. J. Appl. Physiol. 112, 1268-1278. doi: 10.1152/japplphysiol.01305.2011 
Thom, S. R., Yang, M., Bhopale, V. M., Huang, S., and Milovanova, T. N. (2011). Microparticles initiate decompression-induced neutrophil activation and subsequent vascular injuries. J. Appl. Physiol. 110, 340-351. doi: 10.1152/ japplphysiol.00811.2010

Thom, S. R., Yang, M., Bhopale, V. M., Milovanova, T. N., Bogush, M., and Buerk, D. G. (2013). Intramicroparticle nitrogen dioxide is a bubble nucleation site leading to decompression-induced neutrophil activation and vascular injury. J. Appl. Physiol. 114, 550-558. doi: 10.1152/japplphysiol.01386. 2012

Yang, M., Milovanova, T. N., Bogush, M., Uzun, G., Bhopale, V. M., and Thom, S. R. (2012). Microparticle enlargement and altered surface proteins after air decompression are associated with inflammatory vascular injuries. J. Appl. Physiol. 112, 204-211. doi: 10.1152/japplphysiol.00953. 2011

Yu, X., Xu, J., Huang, G., Zhang, K., Qing, L., Liu, W., et al. (2017). BubbleInduced endothelial microparticles promote endothelial dysfunction. PLoS One 12:e0168881. doi: 10.1371/journal.pone.0168881

Zhang, K., Jiang, Z., Ning, X., Yu, X., Xu, J., Buzzacott, P., et al. (2017a). Endothelial-targeting protection by escin in decompression sickness rats. Sci. Rep. 7:41288. doi: 10.1038/srep41288

Zhang, K., Wang, M., Wang, H., Liu, Y., Buzzacott, P., and Xu, W. (2017b). Time course of endothelial dysfunction induced by decompression bubbles in rats. Front. Physiol. 8:181. doi: 10.3389/fphys.2017. 00181

Zhang, K., Wang, D., Jiang, Z., Ning, X., Buzzacott, P., and Xu, W. (2016). Endothelial dysfunction correlates with decompression bubbles in rats. Sci. Rep. 6:33390. doi: 10.1038/srep33390

Conflict of Interest: The author declares that the research was conducted in the absence of any commercial or financial relationships that could be construed as a potential conflict of interest.

Publisher's Note: All claims expressed in this article are solely those of the authors and do not necessarily represent those of their affiliated organizations, or those of the publisher, the editors and the reviewers. Any product that may be evaluated in this article, or claim that may be made by its manufacturer, is not guaranteed or endorsed by the publisher.

Copyright (c) 2021 Arieli. This is an open-access article distributed under the terms of the Creative Commons Attribution License (CC BY). The use, distribution or reproduction in other forums is permitted, provided the original author $(s)$ and the copyright owner(s) are credited and that the original publication in this journal is cited, in accordance with accepted academic practice. No use, distribution or reproduction is permitted which does not comply with these terms. 